\title{
Quantitative phase imaging with partially coherent illumination
}

\author{
T. H. Nguyen, ${ }^{1}$ C. Edwards, ${ }^{2}$ L. L. Goddard, ${ }^{2}$ and G. Popescu ${ }^{1, *}$ \\ ${ }^{1}$ Quantitative Light Imaging Laboratory, Department of Electrical and Computer Engineering, Beckman Institute \\ of Advanced Science and Technology, University of Illinois at Urbana-Champaign, Urbana, Illinois 61801, USA \\ ${ }^{2}$ Micro and Nanotechnology Laboratory, Department of Electrical and Computer Engineering, \\ University of Illinois at Urbana-Champaign, Urbana, Illinois 61801, USA \\ ${ }^{\star}$ Corresponding author: gpopescu@illinois.edu
}

Received July 17, 2014; accepted August 14, 2014;

posted August 20, 2014 (Doc. ID 217093); published September 17, 2014

\begin{abstract}
In this Letter, we formulate a mathematical model for predicting experimental outcomes in quantitative phase imaging (QPI) when the illumination field is partially spatially coherent. We derive formulae that apply to QPI and discuss expected results for two classes of QPI experiments: common path and traditional interferometry, under varying degrees of spatial coherence. In particular, our results describe the physical relationship between the spatial coherence of the illuminating field and the halo effect, which is well known in phase-contrast microscopy. We performed experiments relevant to this common situation and found that our theory is in excellent agreement with the data. With this new understanding of the effects of spatial coherence, our formulae offer an avenue for removing halo artifacts from phase images. (c) 2014 Optical Society of America

OCIS codes: (030.1640) Coherence; (110.0180) Microscopy; (030.0030) Coherence and statistical optics; (100.5070) Phase retrieval.

http://dx.doi.org/10.1364/OL.39.005511
\end{abstract}

Recently, quantitative phase imaging (QPI) has enabled new research areas thanks to its ability to quantify the optical path length fluctuations across transparent specimens [1] . Measuring the complex transmission function associated with a transparent object enables new studies in both materials (see [2-4] and references therein) and life sciences [5-12]. A number of methods have been proposed for $\mathrm{QPI}$ (see [1] and references therein). The common principle of these methods is optical interferometry, in which a reference field, $U_{r}(\mathbf{r})$, is superposed with a sample field, $U_{s}(\mathbf{r})$, with $\mathbf{r}=(x, y)$ being the $2 \mathrm{D}$ spatial coordinate within the image plane. Depending on the way the reference field, $U_{r}$, is obtained, we can separate QPI methods into two groups: (1) traditional interferometry and (2) common path interferometry. In the first group [Fig. 1(a)], the reference field $U_{r}(\mathbf{r}, t)$ is identical to the illumination field, $U_{i}(\mathbf{r}, t)$, carrying no sample information. In this case, the sample field is the illuminated field modulated by the sample, $U_{s}(\mathbf{r}, t)=U_{i}(\mathbf{r}, t) T(\mathbf{r})$, where $T$ is the transmission function of the object, generally complex. Throughout this manuscript, we assume dispersion-free specimens, such that $T$ is independent of the frequency $\omega$. Note that in QPI, the quantity of interest is the phase of this transmission function, $\phi(\mathbf{r})=$ $\arg [T(\mathbf{r})]$. Methods following the traditional principle include digital holographic microscopy $[\underline{1}, 13-15]$, Hilbert phase microscopy (HPM) [16], digitally recorded interference microscopy with automatic phase shifting (DRIMAPS) [17], and optical quadrature microscopy (OQM) [18], etc. On the other hand, in the common path interferometry group, the reference field, $U_{r}(\mathbf{r}, t)$, is derived by spatially filtering the sample field, $U_{s}(\mathbf{r}, t)$. Examples of these methods include Fourier phase microscopy (FPM) [19], diffraction phase microscopy (DPM) [2,20,21], and spatial light interference microscopy (SLIM) $[\underline{10}, \underline{22}]$.

Note that, although a qualitative method, phase contrast microscopy is a form of common-path interferometry [1]. A particular case of common path interferometry for QPI is based on using the transport of intensity equation [23]. Sometimes this method is referred to as "non-interferometric" because it relies on measuring several intensity images around the image focus, without a separate reference field. However, the image itself is just a complicated interferogram, as pointed out early on by Abbe [24]. Thus, the phase information in this case is carried by the interference between the scattered and unscattered components of the image field, much like in phase contrast microscopy [25]. Although the transport of the intensity method was initially proposed for deterministic fields [26,27], later it was applied with partially coherent fields [28]. A more rigorous description of these measurements, employing the coherence mode decomposition of stochastic fields, was reported later by Zysk et al. [29].

In this Letter, we study the effects of the spatial coherence on the outcome of QPI measurements in both traditional and common path experiments. The measurable quantity in QPI is the phase of the spatially dependent, temporal cross-correlation function of the sample and reference field, evaluated at zero delay, $\tau=0$, namely

$$
\Gamma_{s, r}(\mathbf{r}, \mathbf{r}, 0)=\left\langle\mathbf{U}_{s}(\mathbf{r}, t) \mathbf{U}_{r}^{*}(\mathbf{r}, t)\right\rangle,
$$

where the angular brackets denote averaging over the time-varying ensemble of the fields. Throughout this Letter, we assume the fields are ergodic and, thus, stationary. Thus, the ensemble averaging in Eq. (1) can be replaced by time averaging. As a result of the generalized WienerKhintchine theorem [30], $\Gamma_{s, r}(\mathbf{r}, \mathbf{r}, \tau)$ is the Fourier transform of the cross-spectral density, $W_{s, r}(\mathbf{r}, \mathbf{r}, \omega)$. Thus, according to the central ordinate theorem (see, e.g., [1] ), Eq. (2) can be written in terms of $W_{s, r}$, as

$$
\Gamma_{s, r}(\mathbf{r}, \mathbf{r}, 0)=\int W_{s, r}(\mathbf{r}, \mathbf{r}, \omega) \mathrm{d} \omega .
$$


a)

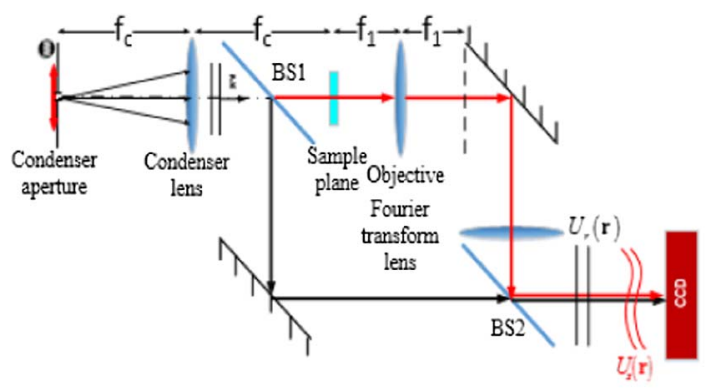

b)

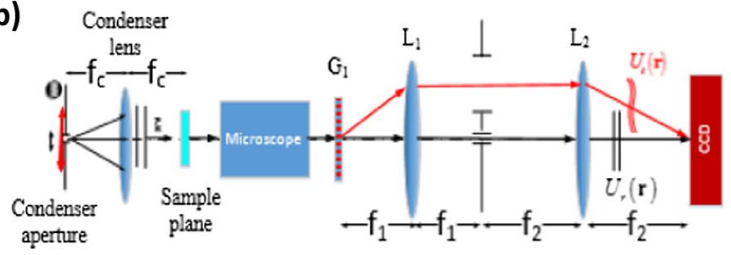

c)

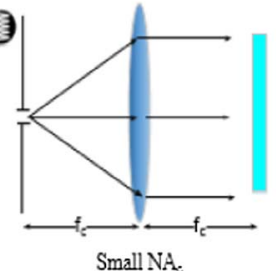

d

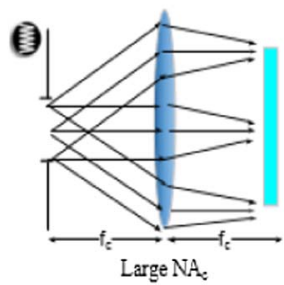

Fig. 1. Traditional and common path QPI configurations. (a) Traditional interferometry: the illumination field $U_{i}(\mathbf{r})$ is split into the sample field, $U_{s}(\mathbf{r})$, and a reference field, $U_{r}(\mathbf{r})$, which interferes at the CCD plane. (b) Common-path interferometry (DPM setup): the sample field is relayed at the output port of the microscope. The reference field $U_{r}(\mathbf{r})$ is derived by spatially filtering the sample field, $U_{s}(\mathbf{r})$. The first-order diffraction pattern generated by a grating $G_{1}$ is used as the sample field at the detector plane. (c) Low numerical aperture of the condenser $\left(\mathrm{NA}_{\text {con }}\right)$ enhances spatial coherence of the illumination field. (d) High $\left(\mathrm{NA}_{\text {con }}\right)$ diminishes the spatial coherence of the illumination field.

Here the cross-spectral density is defined as by $W_{s, r}(\mathbf{r}, \mathbf{r}, \omega)=\left\langle U_{s}(\mathbf{r}, \omega) U_{r}^{*}(\mathbf{r}, \omega)\right\rangle$ where $U_{s}(\mathbf{r}, \omega)$ and $U_{r}(\mathbf{r}, \omega)$ are the Fourier transforms of $U_{s}(\mathbf{r}, t)$ and $U_{r}(\mathbf{r}, t)$, respectively. Again, the angular bracket denotes the ensemble averaging. We now derive the specific forms of Eq. (1) for traditional and common-path interferometry and describe how spatial coherence affects accurate retrieval of the object function $T(\mathbf{r})$.

Traditional interferometry. Let us consider the case where the reference field is simply a replica of the illumination field, $U_{r}(\mathbf{r}, \omega)=U_{i}(\mathbf{r}, \omega)$. The sample field is given as $U_{s}(\mathbf{r}, \omega)=T(\mathbf{r}) U_{i}(\mathbf{r}, \omega)$. Modalities falling into this categories include, but are not limited to [31, 15$]$ and [32]. For such cases, Eq. (1) becomes

$$
\Gamma_{s, r}(\mathbf{r}, \mathbf{r}, 0)=T(\mathbf{r}) \int S_{i}(\mathbf{r}, \omega) \mathrm{d} \omega .
$$

Here, $S_{i}(\mathbf{r}, \omega)=W_{i}(\mathbf{r}, \mathbf{r}, \omega)$ is the spatially dependent power spectrum. Since the second factor on the right hand side of Eq. (3) is a positive quantity, it follows that the measured phase, $\phi_{m}(\mathbf{r})=\arg \left[\Gamma_{s, r}(\mathbf{r}, \mathbf{r}, 0)\right]$, is identical

with the phase of interest, $\phi(\mathbf{r})=\arg [T(\mathbf{r})]$. Therefore, QPI measurements can be performed accurately regardless of the spatial coherence of the illumination, as long as the reference and illumination fields are identical. Note that obtaining the cross-correlation function $\Gamma_{s, r}$ experimentally involves introducing controllable phase difference between the two fields, either in space or time [see, e.g., Chap. 8 in Ref. (1)].

Common-path interferometry. Next, let us consider the case when the reference field is derived by spatially filtering the sample field via, for example, passing the central portion of its Fourier transform through a small pinhole [Fig. 1(b)]. Denoting the spatial filtering kernel characterizing this derivation by $h_{o}(\mathbf{r})$, the reference field can be written in explicit forms as

$$
U_{r}(\mathbf{r}, \omega)=U_{s}(\mathbf{r}, \omega) \bigodot_{\mathbf{r}} h_{o}(\mathbf{r}),
$$

where $\mathrm{V}_{\mathbf{r}}$ denotes the two-dimensional convolution operator. In this case, the temporal cross-correlation function is obtained by combining Eqs. (2) and (4), namely

$$
\Gamma_{s, r}(\mathbf{r}, \mathbf{r}, 0)=T(\mathbf{r}) \int\left[\int W_{i}\left(\mathbf{r}, \mathbf{r}^{\prime}, \omega\right) h_{o}^{*}\left(\mathbf{r}-\mathbf{r}^{\prime}\right) T^{*}\left(\mathbf{r}^{\prime}\right) \mathrm{d}^{2} \mathbf{r}^{\prime}\right] \mathrm{d} \omega .
$$

Note that the quantity between square brackets in Eq. (5), $W_{i}\left(\mathbf{r}, \mathbf{r}^{\prime}, \omega\right)$, is the cross-spectral density of the illumination field at the sample plane. Let us consider that the illumination field is statistically homogeneous, i.e., $W_{i}\left(\mathbf{r}, \mathbf{r}^{\prime}, \omega\right)=W_{i}\left(\mathbf{r}-\mathbf{r}^{\prime}, \omega\right)$ such that the expression for the cross-correlation function becomes

$$
\begin{aligned}
\Gamma_{s, r}(\mathbf{r}, \mathbf{r}, 0) & =T(\mathbf{r}) \int\left[\int W_{i}\left(\mathbf{r}, \mathbf{r}^{\prime}, \omega\right) \mathrm{d} \omega\right] h_{o}^{*}\left(\mathbf{r}-\mathbf{r}^{\prime}\right) T^{*}\left(\mathbf{r}^{\prime}\right) \mathrm{d}^{2} \mathbf{r}^{\prime} \\
& =T(\mathbf{r}) \int \Gamma_{i}\left(\mathbf{r}-\mathbf{r}^{\prime}, 0\right) h_{o}^{*}\left(\mathbf{r}-\mathbf{r}^{\prime}\right) T^{*}\left(\mathbf{r}^{\prime}\right) \mathrm{d}^{2} \mathbf{r}^{\prime} \\
& =T(\mathbf{r})\left[T(\mathbf{r}) \bigotimes_{\mathbf{r}} h(\mathbf{r})\right]^{*}
\end{aligned}
$$

where we have defined $h(\mathbf{r})=\Gamma_{i}^{*}(\mathbf{r}, 0) h_{o}(\mathbf{r})$. Equation (ㅁ) establishes a relationship between the measurable quantity in QPI, $\Gamma_{s, r}$, and the transmission function of the object, $T$, when: (1) the illuminating filed is spatially partially coherent and characterized by the correlation function, $\Gamma_{i}(\mathbf{r}, 0)$, and (2) the reference field is obtained by blurring the sample field via a kernel $h_{o}(\mathbf{r})$. As a side note, expanding the condenser aperture of the illumination reduces the degree of coherence for the illumination, i.e., $\Gamma_{i}$ becomes narrower and vice versa. In contrast to the previous case, now the measurable quantity, $\Gamma_{s, r}(\mathbf{r}, \mathbf{r}, 0)$, is no longer just the transmission function $T(\mathbf{r})$, multiplied by a positive function. The measured phase, $\phi_{m}$, differs from the expected value, specifically

$$
\phi_{m}(\mathbf{r})=\phi(\mathbf{r})-\arg \left[\left(T \bigodot_{\mathbf{r}} h\right)(\mathbf{r})\right] .
$$

Equation (7) is the key result of our Letter. It indicates that the measured phase at each point in the field of view depends on the phase at neighboring points, which is the result of the convolution with the kernel $h(\mathbf{r})$. It is 
physically insightful to discuss two asymptotic cases: (i) extremely narrow $h$, i.e., $h(\mathbf{r}) \rightarrow \delta(\mathbf{r})$ and (ii) extremely broad $h$, i.e., $h(\mathbf{r}) \approx$ const. The first case applies when the illumination field is completely incoherent, which results in a kernel $h$ much narrower than function $T$. Consequently, $\left(T \S_{\mathrm{r}} h\right)(\mathbf{r}) \rightarrow T(\mathbf{r})$, and the measured phase vanishes,

$$
\phi_{m}^{\text {incoh }}(\mathbf{r}) \approx 0 .
$$

Thus, Eq. (8) establishes the impossibility of measuring any phase information using spatially incoherent light, even when the spatial filter used to render the reference field is perfectly closed, i.e., $h_{o}=$ const. By contrast, if the illumination field is perfectly coherent, $W_{i}\left(\mathbf{r}-\mathbf{r}^{\prime}\right) \approx$ const, $\forall \mathbf{r}, \mathbf{r}^{\prime}$, and, in addition, the spatial filtering is perfect ( $h_{o}=$ const.), $h$ becomes much broader than the object function, $T(\mathbf{r})$. In this case, we have $\left(T \bigodot_{\mathbf{r}} h\right)(\mathbf{r}) \approx$ const., which results in an accurate measurement,

$$
\phi_{m}^{\mathrm{coh}}(\mathbf{r})=\phi(\mathbf{r}) .
$$

All situations of experimental relevance exist between the two extreme cases of complete coherence and complete incoherence, described by Eq. () and (9), respectively. When the coherence area of the illumination field is comparable with the size of the object of interest, the measured phase map misses the low frequencies that are contained in the function $T \mathrm{\vee}_{\mathrm{r}} h$. This artifact is well known as the "halo effect" in phase contrast microscopy $[\underline{25}, 33]$.

Next, we show a comparison between experimental measurements and our theoretical prediction. Our measurements were performed using DPM [Fig. 1(b)] with different values of the condenser numerical aperture, $\mathrm{NA}_{\text {con }}$ ). More details about the setup and obtaining the correlation function, $\Gamma_{s, r}(\mathbf{r}, \mathbf{r}, 0)$ from the interference pattern, can be found elsewhere, e.g., [34]. For this experiment, transparent quartz micropillars were fabricated with known dimensions to serve as control samples. A 1" quartz wafer was patterned using SPR 511a positive photoresist and transferred to the quartz substrate by etching in a reactive-ion etcher (RIE) using a CF4 (Freon 14) plasma, resulting in square micropillars of various widths and a height of $123 \mathrm{~nm}$, as measured by the Alpha Step IQ Profilometer. The refractive index of quartz used in the simulation was 1.545 , and the center wavelength of the source was $574 \mathrm{~nm}$ as measured experimentally [35]. We obtained the phase maps and topography associated with $5,10,20$, and $40 \mu \mathrm{m}$ width pillars.

Figures 2 and 3 show a comparison between our theoretical model and experimental measurements taken using DPM. In order to model the effects of spatial coherence on QPI measurements, calculations based on Eq. (7) were performed in MATLAB for the case of monochromatic light. In our calculations, the DPM pinhole filter was assumed to be perfect, i.e., $h_{o}(\mathbf{r}) \approx$ const which is a good approximation when using a $10-\mu \mathrm{m}$ pinhole under the current DPM configuration [35]. The spatial power spectrum associated with the condenser aperture is approximated very well by a Gaussian function (see inset

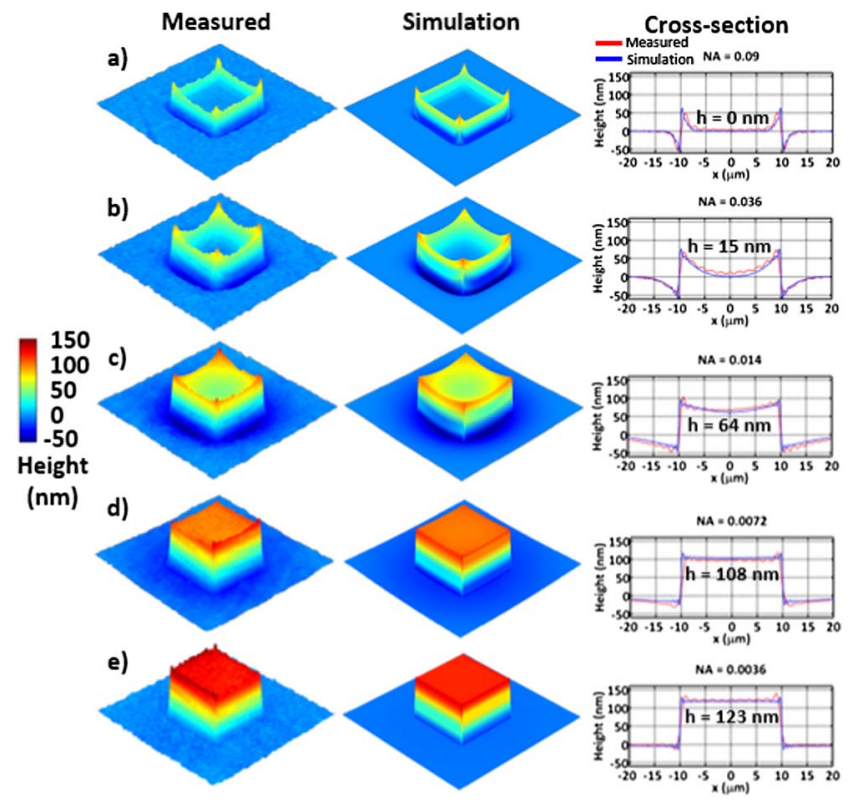

Fig. 2. Comparison between measured and calculated profiles for quartz pillars, as described in text. The $\mathrm{NA}_{\text {con }}$ values are decreasing from (a) to (e), as shown above each topography profile.

of Fig. 3). Clearly, our calculation predicts accurately the phase reduction under partially coherent illumination. Furthermore, our model also accurately describes artifacts in the measured topography, i.e., the halos commonly observed in QPI and phase contrast microscopy $[1,35]$. Notice that opening up the condenser aperture reduces the spatial coherence to the point where only edges of the pillar are reconstructed in the phase image. However, using smaller pinholes for spatially filtering the illumination light results in increasingly accurate phase and topography measurement, as summarized in Fig. 3. In other words, as the coherence area is increased, the halo diminishes and the height map converges to the ground truth.

In sum, we presented a quantitative description of the role of spatial coherence in QPI for both commonpath and noncommon-path configurations. This model

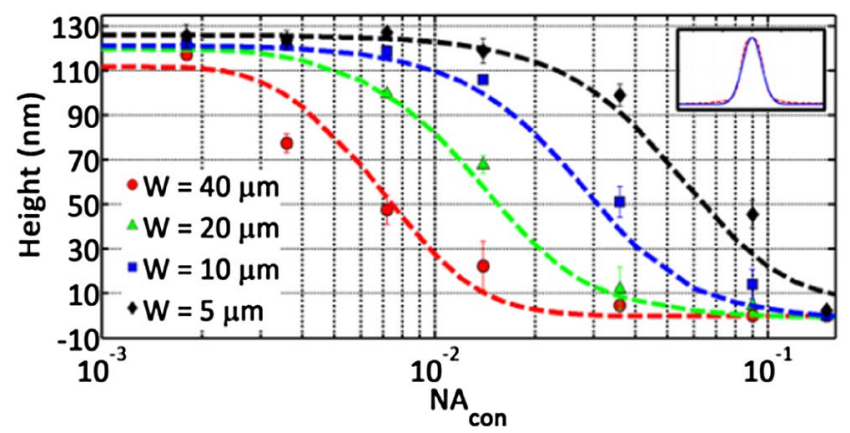

Fig. 3. Comparison between measured (markers) and calculated [Eq. (7)] pillar height values (lines) versus numerical aperture of the condenser $\mathrm{NA}_{\text {con }}$ for 4 different pillars. The inset compares the normalized intensity measured from the condenser aperture as a function of spatial frequency in $\mathrm{rad} / \mu \mathrm{m}$ for a $200 \mu \mathrm{m}$ pinhole $\left(\mathrm{NA}_{\mathrm{con}}=0.0072\right)$ to a Gaussian fit function with full width at half-maximum (FWHM) of $0.158 \mathrm{rad} / \mu \mathrm{m}$. 
explains the presence of the halo effect and the observed reduction in measured height. Our formulas are expected to set a foundation for further work in the correction of aberrations and phase inaccuracies in partially coherent imaging systems.

This work was supported by the National Science Foundation CBET-1040461 MRI with matching funds from the University of Illinois and by Agilent Laboratories. For more information, visit http://light.ece.illinois.edu/.

\section{References}

1. G. Popescu, Quantitative Phase Imaging of Cells and Tissues (Mcgraw-Hill, 2011).

2. C. Edwards, A. Arbabi, G. Popescu, and L. L. Goddard, Light Sci. Appl. 1, e30 (2012).

3. R. Zhou, C. Edwards, A. Arbabi, G. Popescu, and L. L. Goddard, Nano Lett. 13, 3716 (2013).

4. C. Edwards, K. Wang, R. Zhou, B. Bhaduri, G. Popescu, and L. L. Goddard, Opt. Express 21, 13547 (2013).

5. H. V. Pham, B. Bhaduri, K. Tangella, C. Best-Popescu, and G. Popescu, Plos One 8, e55676 (2013).

6. M. Mir, Z. Wang, Z. Shen, M. Bednarz, R. Bashir, I. Golding, S. G. Prasanth, and G. Popescu, Proc. Natl. Acad. Sci. 108, 13124 (2011).

7. Y. K. Park, C. A. Best, K. Badizadegan, R. R. Dasari, M. S. Feld, T. Kuriabova, M. L. Henle, A. J. Levine, and G. Popescu, Proc. Natl. Acad. Sci. 107, 6731 (2010).

8. Y. K. Park, M. Diez-Silva, G. Popescu, G. Lykotrafitis, W. Choi, M. S. Feld, and S. Suresh, Proc. Natl. Acad. Sci. USA 105, 13730 (2008).

9. H. F. Ding, Z. Wang, F. Nguyen, S. A. Boppart, and G. Popescu, Phys. Rev. Lett. 101, 238102 (2008).

10. T. H. Nguyen and G. Popescu, Biomed. Opt. Express 4, 1571 (2013).

11. K. Creath and G. Goldstein, Biomed. Opt. Express 3, 2866 (2012).

12. P. Bon, G. Maucort, B. Wattellier, and S. Monneret, Opt. Express 17, 13080 (2009).

13. P. Marquet, B. Rappaz, P. J. Magistretti, E. Cuche, Y. Emery, T. Colomb, and C. Depeursinge, Opt. Lett. 30, 468 (2005).
14. W. M. Ash and M. K. Kim, Opt. Express 16, 9811 (2008).

15. F. Dubois, N. Callens, C. Yourassowsky, M. Hoyos, P. Kurowski, and O. Monnom, Appl. Opt. 45, 864 (2006).

16. T. Ikeda, G. Popescu, R. R. Dasari, and M. S. Feld, Opt. Lett. 30, 1165 (2005).

17. D. Zicha and G. A. Dunn, J. Microsc. 179, 11 (1995).

18. D. Hogenboom, C. A. DiMarzio, T. J. Gaudette, A. J. Devaney, and S. C. Lindberg, Opt. Lett. 23, 783 (1998).

19. G. Popescu, L. P. Deflores, J. C. Vaughan, K. Badizadegan, H. Iwai, R. R. Dasari, and M. S. Feld, Opt. Lett. 29, 2503 (2004).

20. G. Popescu, T. Ikeda, R. R. Dasari, and M. S. Feld, Opt. Lett. 31, 775 (2006).

21. B. Bhaduri, H. Pham, M. Mir, and G. Popescu, Opt. Lett. 37, 1094 (2012).

22. Z. Wang, L. J. Millet, M. Mir, H. Ding, S. Unarunotai, J. A. Rogers, M. U. Gillette, and G. Popescu, Opt. Express 19, 1016 (2011).

23. A. Barty, K. A. Nugent, D. Paganin, and A. Roberts, Opt. Lett. 23, 817 (1998).

24. E. Abbe, Arch. Mikrosk. Anat. 9, 431 (1873).

25. F. Zernike, Science 121, 345 (1955).

26. M. R. Teague, J. Opt. Soc. Am. 73, 1434 (1983).

27. T. E. Gureyev and K. A. Nugent, Opt. Commun. 133, 339 (1997).

28. D. Paganin and K. A. Nugent, Phys. Rev. Lett. 80, 2586 (1998).

29. A. M. Zysk, R. W. Schoonover, P. S. Carney, and M. A. Anastasio, Opt. Lett. 35, 2239 (2010).

30. L. Mandel and E. Wolf, Optical Coherence and Quantum Optics (Cambridge University, 1995).

31. M. Somekh, C. See, and J. Goh, Opt. Commun. 174, 75 (2000).

32. Y. Park, W. Choi, Z. Yaqoob, R. Dasari, K. Badizadegan, and M. S. Feld, Opt. Express 17, 12285 (2009).

33. T. Wilson and C. J. R. Sheppard, Optik 59, 19 (1981).

34. B. Bhaduri, C. Edwards, H. Pham, R. Zhou, T. Nguyen, L. Goddard, and G. Popescu, Adv. Opt. Photon. 6, 57 (2014).

35. C. Edwards, B. Bhaduri, T. Nguyen, B. G. Griffin, H. Pham, T. Kim, G. Popescu, and L. L. Goddard, Opt. Express 22, 5133 (2014). 